ELs are rare benign tumors of the bronchial tree; only 80 cases have been previously reported in the English literature. Most ELs arise in the submucosal layer of the bronchus; fatty tissue is normally found in both the mucosa and the tissue external to the cartilage. Almost two thirds of the tumors occur in the right side, and they are usually located in the main-stem or lobar bronchi. ${ }^{1-3}$ Smoking and obesity are significant risk factors for EL development. ${ }^{4}$ Cough, hemoptysis, and shortness of breath are common symptoms, ${ }^{4,5}$ and patients are often misdiagnosed and treated for asthma for many years, as was our patient.

Owing to the endobronchial tumor growth, irreversible changes in distal pulmonary parenchyma often occur: atelectasis, overinfection, pneumonia, and fibrosis are frequently observed. Bronchoscopy is necessary to identify and locate the lesion and to differentiate it from a malignant tumor. Differential pathologic diagnosis between bronchial carcinoid and lipoma is often difficult because of the paucity of tissue sampling at biopsy. The tumor's surface is usually paler in the lipoma and more friable in the carcinoid. EL sometimes coexists with lung cancer, especially in patients who have smoked heavily. ${ }^{1}$

Bronchoscopic resection should be considered the first choice of treatment for EL; Muraoka and associates ${ }^{2}$ report that no patient presented with bronchial tumor recurrence after EL bronchoscopic resection. Tumor surgical resection is preferred in some cases: (1) extrabronchial growth or subpleural lipomatous disease; (2) expected technical difficulties during the bronchoscopic resection; (3) possible malignant tumor coexisting with EL; and (4) peripheral irreversible lung parenchyma changes after long-term atelectasis or pneumonia.

In conclusion, EL is a rare benign endobronchial tumor that can be safely resected by a bronchoscopic procedure. Surgery is recommanded when the distal lung parenchyma is damaged by an obstructive pneumonia or fibrosis.

\section{References}

1. Kamiyoshihara M, Sakata K, Otani Y, Kawashima O, Takahashi T, Morishita Y. Endobronchial lipoma accompained with primary lung cancer: report of a case. SurgToday. 2002;32:402-5.

2. Muraoka M, Oka T, Akamine S, Nagayasu T, Iseki M, Suyama N, et al. Endobronchial lipoma. Chest. 2003;123:293-6.

3. Jensen MS, Petersen AH. Bronchial lipoma: three cases and review of the literature. Scand J Thorac Cardiovasc Surg. 1970;4:131-4.

4. Schraufnagel DE, Morin JE, Wang NS. Endobronchial lipoma. Chest. 1979;75:97-9.

5. Politis J, Funahashi A, Gehlsen JA, DeCock D, Stengel BF, Choi H. Intrathoracic lipomas: report of three cases and review of the literature with the emphasis on endobronchial lipoma. $J$ Thorac Cardiovasc Surg. 1979;77:550-6.

\title{
A new type of diaphragmatic hernia: Anterolateral hernia
}

\author{
Jérôme Mouroux, MD, PhD, Daniel Pop, MD, Patrice Guiraudet, MD, Ricardo Giovanetti, MD, Jérôme Lauron, MD, \\ and Nicolas Venissac, MD, Nice, France
}

$\mathrm{H}$ eterogenous types of acquired hernias in the diaphragm have been described in the literature: the hiatal hernia through the esophageal foramen and the posterolateral hernia through the Henle costolumbar orifice or anterior foramen of Larrey-Morgagni hernia (retroxiphoid hernias). ${ }^{1,2}$ To our knowledge, no anterolateral hernia has been reported. We describe the case of a hernia between the eighth and ninth anterior costochondral insertion of the muscular fibers of the diaphragm.

From the Thoracic Surgery Department, Pasteur Hospital, Nice, France. Received for publication Sept 14, 2007; accepted for publication Dec 4, 2007.

Address for reprints: Daniel Pop, MD, Thoracic Surgery Department, Pasteur Hospital, Buiding H1, 30 Avenue de la Voie Romaine, 06002 Nice, France (E-mail: danielpopch@yahoo.com).

J Thorac Cardiovasc Surg 2008;135:955-6

$0022-5223 / \$ 34.00$

Copyright (C) 2008 by The American Association for Thoracic Surgery doi:10.1016/j.jtcvs.2007.12.001

\section{Clinical Summary}

A 76-year-old man had a medical history of diabetes mellitus, arterial hypertension, a bilateral inguinal hernia (operated), and a $2 / 3$ gastrectomy for gastric ulcer (50 years ago). In 1999, a chest xray showed an air-filled opacity in the left thoracic base. No further investigations were done. In September of 2005, the patient experienced a blocking respiration symptom in the left hemithorax, especially after meals and occasionally associated with vomiting. The chest $\mathrm{x}$-ray showed colic haustration at the left thoracic base. A gastroesophageal endoscopy showed no anomaly. The cardial sphincter was $40 \mathrm{~cm}$ from the incisor teeth, and the gastric stump and duodenal-gastric anastomosis were healthy. The barium esophagography showed a normal cardial region. The chest computed tomography scan demonstrated a colic ascent in the left thorax and the small intestine through an anterolateral hole of the diaphragm (Figure 1).

Surgery was performed via a low left posterolateral thoracotomy. We found the peritoneal sac containing the digestive loops (Figure 2). After clearly identifying the edges, we resected and closed the sac. These edges corresponded to the muscular fibers of the diaphragm inserted at the eighth and ninth chondrocostal cartilage. The hole measured $10 \mathrm{~cm}$ in the anteroposterior diameter, was located far from the retroxiphoid region, and ended at the central tendon. The closure was done with nonabsorbable sutures. The 


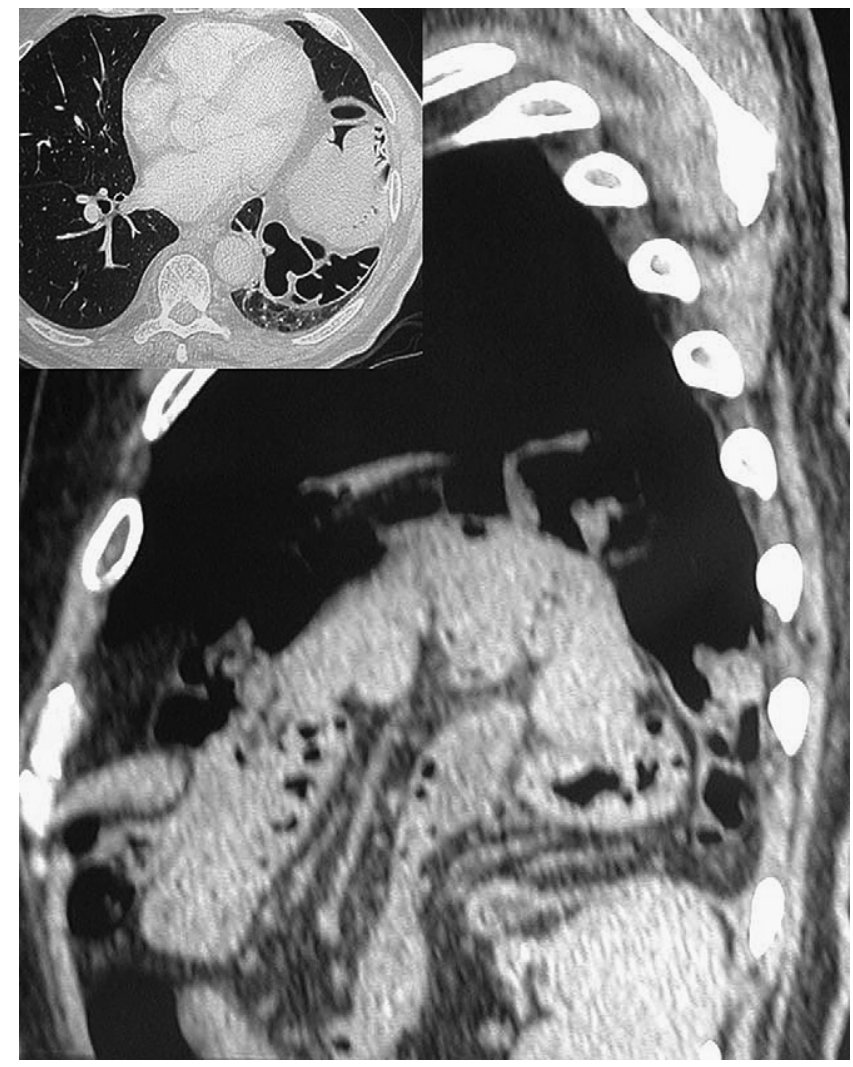

Figure 1. Saggital computed tomographic scan showing the diaphragmatic hernia; transversal slice in frame.

postoperative period was uneventful, and the follow-up was marked by a normal chest $\mathrm{x}$-ray and the disappearance of symptoms.

\section{Conclusions}

There are two anatomic types of classic retroxiphoid hernias: The first type is located in a median weakness area situated between the sternal muscular fibers of the diaphragm, also known as Marfan's foramen. The second type is located between the muscular fibers of the xiphisternum and the costal margins traversed by the internal thoracic artery (that becomes the superior epigastric artery), also known as Larrey's space or Morgagni's foramen. ${ }^{1,2}$ Most authors think that the lack of fusion or muscularization of the pleuroperitoneal membrane leads to a defect in the costosternal trigones. ${ }^{3,4}$ In $90 \%$ of cases, retroxiphoid hernias are located on the right side. ${ }^{1}$ We observed a left anterolateral hernia outside these weakness points of the diaphragm. Laterally, from an anatomic point of view, the muscular fibers of the diaphragm extend from the central tendon to the rib cage, where they are mixed with the insertions of the abdominal transverse muscle. Each insertion allows the passage of the intercostal pedicle. ${ }^{5}$ We suppose that this area of passage can

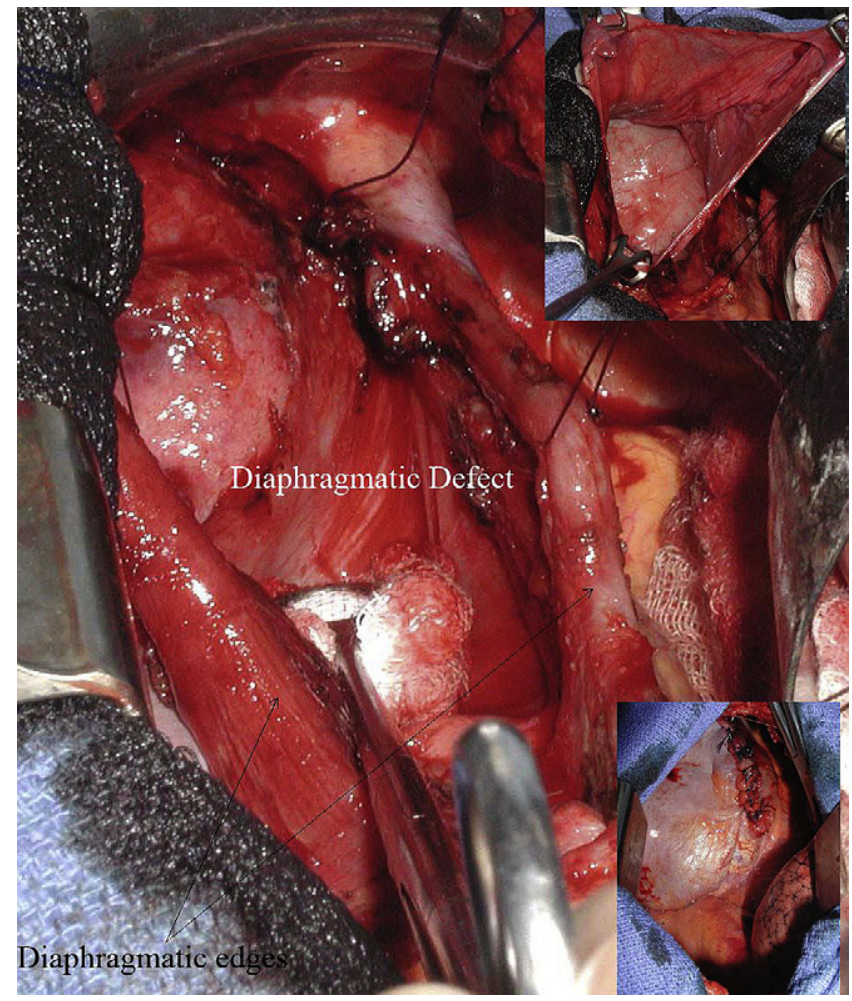

Figure 2. Perioperative view with the muscular fibers of the diaphragm (peritoneal sac resected). Peritoneal sac and colon (upper right). The defect is repaired (lower right).

be exposed to weakening. Our patient had no trauma in his medical history, and the prior gastric surgery (via median laparotomy) is an unlikely cause because it was performed several years ago.

This anterolateral hernia must be considered as a "weakness" hernia, similar to the inguinal hernia the patient previously had. The surgical treatment of this hernia was correct because of the potential life-threatening complications similar to the other types of diaphragmatic hernias.

\section{References}

1. Comer TP, Clagett OT. Surgical treatment of hernia of the foramen of Morgagni. J Thorac Cardiovasc Surg. 1966;52:461-8.

2. Mouroux J, Venissac N, Alifano M, Padovani B. Morgagni hernia and thoracic deformities. Thorac Cardiovasc Surg. 2003;51:44-5.

3. Kelly KA, Bassett DL. An anatomic reappraisal of the hernia of Morgagni. Surgery. 1964;55:495-9.

4. Shumpelick V, Steinau G, Schulper J, Prescher A. Surgical embryology and anatomy of the diaphragm with surgical application. Surg Clin N Am. 2000;80:213-39.

5. Rouvière H. Anatomie Humaine, 10th Edition. Paris: Masson \& Co; 1967:87-92. 